\title{
Atividades fisioterapêuticas em grupos para idosos institucionalizados: a percepção do idoso
}

Fisioterapeutica activities in groups

for elderly institutionalized: perception

of the elderly

FisiSenectus. Unochapecó Ano 5, n. 2 - Jul/Dez. 2017 p. $47-53$

José Nilson Rodrigues de Menezes. nilson.pesquisa@hotmail.com

Fisioterapeuta, Mestre em Educação em Saúde pela Universidade de Fortaleza (UNIFOR). Doutor em Biotecnologia pela Universidade Estadual do Ceará (UECE). Docente da Universidade de Fortaleza (UNIFOR).

Francisco Jenilson de Sousa Costa. jenilson.sousa18@gmail.com

Fisioterapeuta. Especialista em andamento em Fisioterapia Traumo-Ortopedica e Desportiva pelo Centro Universitário Christus (UNICHRISTUS).

Anna Karla Ribeiro Lima. karla_rlimag@hotmail.com Fisioterapeuta pela Universidade de Fortaleza (UNIFOR).

Cleidiane Gonzaga Damasceno de Souza. cleidiane.gonzaga28@yahoo.com.br Graduanda em Fisioterapia pela Universidade de Fortaleza (UNIFOR).

Liliana Gama Oliveira. lilihannag@hotmail.com

Graduanda em Fisioterapia pela Universidade de Fortaleza (UNIFOR).

Ranieli Cavalcante dos Santos. raniellycavalcante@hotmail.com

Graduanda em Fisioterapia pela Universidade de Fortaleza (UNIFOR).

\section{Resumo}

Introdução: 0 envelhecimento é caracterizado como desenvolvimento social, com características ao longo de todo o percurso da existência, um processo ativo e progressivo, no qual sucedem alterações morfológicas, funcionais, e psicológicas que geram, perda da capacidade do indivíduo Objetivo: Identificar a percepção do idoso institucionalizado sobre a contribuição das atividades fisioterapêutica em grupos. Materiais e Métodos: Realizou-se um estudo descritivo de natureza qualitativa com idosos. Participaram da pesquisa idosos com idade igual ou superior a 60 anos do gênero masculino e feminino que já realizavam atendimento fisioterapêutico, residentes na Unidade de Abrigo de Idosos, no período de fevereiro a março de 2016. Foram excluídos da pesquisa pacientes com dificuldades na fala ou alterações no estado mental que inviabilizassem a entrevista. As informações foram coletadas através de uma entrevista semiestruturada, e foram analisadas pela técnica de análise temática. Está pesquisa foi aprovada pelo Comitê de Ética em Pesquisa da Universidade de Fortaleza (COÉTICA-UNIFOR), com o parecer no 1.346.007. Resultados: Os idosos relatam que as atividades fisioterapêuticas contribuíram para a melhoria da força, do equilíbrio, da marcha, sabendo-se que a falta de exercícios diários tem como principais consequências limitações físicas, emocionais e sociais, e que a atividade fisioterapêutica trouxe uma melhoria considerável facilitando a melhoria dos seus movimentos durante os exercícios, do estado psíquico, e favorecendo uma melhor relação entre si, tornando as atividades

\section{Fisißenectus}


mais prazerosas durante a Fisioterapia. Conclusão: Conclui-se que os idosos perceberam a efetividade da Fisioterapia por meio das atividades fisioterapêuticas em grupos, visto que melhorou seu estado funcional, psíquico e social.

\title{
Palavras-chave
}

Idoso; Fisioterapia; Tratamento.

\begin{abstract}
Introduction: Aging is characterized as social development, with characteristics throughout the course of existence an active and progressive process, in which morphological, functional, and psychological changes that generate, loss of the individual's capacity Objective: To identify the perception of the institutionalized elderly about the contribution of Physical Therapy activities in groups. Materials and methods: A descriptive study of a qualitative nature was carried out with the elderly. Have been in the research elderly aged about 60 years old or over of the male and female gender who have already performed physiotherapeutic care, residents of the Shelter Unit for the Elderly in the period from February to March 2016. Patients with speech difficulties or changes in mental status that make interview impossible were excluded from the study. The information was collected by a semi-structured interview, and was analyzed using the thematic analysis technique. This research was approved by the Ethics Committee of the University of Fortaleza (UNIFOR), with opinion No. 1,346,007. Results: The elderly report that physical therapy activities have contributed to the improvement of strength, balance and gait, since the lack of daily exercise has the main consequences of physical, emotional and social limitations, and that the physical therapy activity has brought a considerable improvement in facilitating the improvement of their movements during the exercises, of the psychic state, and favoring a better relation between them, making the activities more pleasurable during Physical Therapy. Conclusion: Therefore, concludes that the elderly realized the effectiveness of Physiotherapy through physical therapy activities in groups improving their functional status, psychological and social.
\end{abstract}

\section{Keywords}

Elderly; Physical Therapy; Treatment.

\section{Introdução}

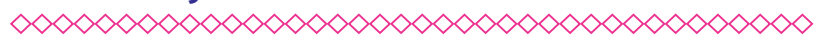

Há cerca de 40 anos, nota-se o crescimento da população idosa nos países em desenvolvimento. E, em nível mundial, há um aumento exponencial, cuja projeção, para o ano de 2050, será de um idoso (com idade igual ou superior a 60 anos) para cada cinco jovens ${ }^{1}$.

O envelhecimento da população em grande parte do mundo tornou-se realidade nas últimas décadas. As mudanças nas condições de vida da população e os avanços da medicina no combate a enfermidades diminuíram as mortalidades precoces e promoveram um aumento dos anos de vida ${ }^{2}$.

A velhice vem acompanhada de um declínio da circunstância de disposição física, mental e social ${ }^{3}$. O maior número de idosos no Brasil e no mundo alavancou as pesquisas sobre envelhecimento.
Apesar da cultura atual ainda impor valor negativo à velhice, estudos têm considerado esse processo como um todo e não apenas como o aumento da idade, abrindo novos horizontes para se viver meIhor essa fase da vida ${ }^{4}$.

0 envelhecimento pode ser caracterizado como um desenvolvimento social, com diferentes características ao longo de todo o percurso da existência ${ }^{5}$. Esse processo é uma evolução continuada e que provoca uma redução progressiva das atividades fisiológicas, o que minimiza a capacidade do organismo, propicia a manifestação de agravos e termina com o fim ${ }^{6}$. As restrições físicas, os danos cognitivos e as doenças crônicas poderão aparecer no transcorrer do envelhecimento ${ }^{7}$. É um processo ativo e progressivo, em que sucedem alterações morfológicas, funcionais, bioquímicas e psicológicas que geram a perda da capacidade de adaptação do indivíduo ao meio ambiente, e causam, dessa forma, maior vulnerabilidade e 
maior incidência de processos patológicos ${ }^{8}$. Uma dessas modificações é a redução da aptidão funcional, de forma geral ${ }^{9}$. Há também a diminuição da capacidade de variação dos reflexos adaptativos, do processamento dos sinais vestibulares, visuais e proprioceptivos que são responsáveis pela permanência do equilíbrio corpora ${ }^{10}$.

Pode-se viver a velhice como uma etapa da vida em que o conhecimento, a sensatez e a tranquilidade traduzem a essência dessa fase ${ }^{11}$. 0 ponto central no envelhecimento é a conservação da capacidade de manter seus projetos pessoais, tendo uma vida com sentido e propósitos até as idades mais avançadas, tanto para quem proporciona o cuidado quanto para quem o recebe ${ }^{12}$.

O envelhecimento vêm acompanhado de uma série de mudanças orgânicas, com declínio das capacidades funcionais e alterações no funcionamento fisiológico de órgãos e sistemas ${ }^{13}$.

Ademais, destaca-se que o idoso institucionalizado representa, em sua maior parte, uma classe privada socialmente, por estar distante da família, do domicílio, dos amigos, dos vínculos sociais ${ }^{14}$. Observa-se, muitas vezes, que o desejo do idoso não é mais importante. E este passa a perder sua autonomia e liberdade ${ }^{15}$. Com isso, ocorrem mudanças nos contextos de vida do indivíduo. Os déficits cognitivos em um idoso são, muitas vezes, a origem de problemas funcionais, fazendo com que haja dano ou dificuldade para adquirir ou manter as aptidões e habilidades ${ }^{16}$.

Nas Instituições de Longa Permanência de Idosos (ILPIs), os profissionais de saúde são preparadospara cuidar dos idosos ${ }^{17}$. As ILPIs buscam preservar a autonomia e a independência do idoso, respeitar a manutenção dos papéis sociais, garantindo seu acesso à saúde e proporcionando um melhor cuidado integral, tendo em vista promover uma melhor qualidade de vida para esses indivíduos, minimizando agravos inevitáveis e restrições que a vida na instituição pode acarretar ${ }^{18}$.

A Fisioterapia tem como objetivo primordial manter esses idosos com o máximo de capacidade funcional, percebendo suas limitações, e de independência física e mental. Logo, a Fisioterapia no idoso atua na promoção da saúde e nas mudanças no estilo de vida dos indivíduos, o que acarretaria uma diminuição dos riscos de eles adoecerem e morrerem ${ }^{19}$.

Envelhecer é inevitável, bem como, conviver com as disfunções que o acompanham.. Nessa direção, deve-se compreender a importância de realizar ações de promoção da saúde na população idosa, buscando a prevenção de enfermidades e complicações ${ }^{20}$.

Importa mencionar que as atividades fisioterapêuticas conseguem reduzir o uso de medicamentos, favorecer a independência do idoso, e, ainda, aumentar o convívio com outros indivíduos fora da instituição acolhedora ${ }^{21}$.

A pesquisa objetivou identificar a percepção do idoso institucionalizado sobre a contribuição das atividades fisioterapêutica em grupos.

\section{Materiais e Métodos}

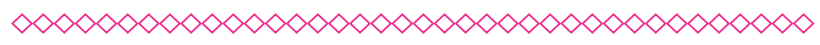

Esta pesquisa foi realizada em uma Unidade de Abrigo de Idosos, localizada na Avenida Olavo Bilac, 1280, no bairro São Gerardo. O local é mantido pelo Governo do Estado do Ceará, por intermédio da Secretaria do Trabalho e Desenvolvimento Social (STDS). Os dados foram coletados no período de fevereiro a março de 2016.

Foram entrevistados idosos do gênero masculino e feminino que residem no Abrigo de Idosos do Ceará. Foram incluídos pacientes idosos que já realizaram atendimento fisioterápico. Foram excluídos pacientes com dificuldades na fala ou alterações no estado mental que inviabilizaram a entrevista.

As informações foram coletadas através de uma entrevista semiestruturada, que foi transcrita. Conforme estudos, este tipo de entrevista favorece que os entrevistados falem sobre o tema em questão sem respostas ou condições determinadas pelo pesquisador. A entrevista foi realizada de maneira individual em um ambiente reservado.

As informações foram analisadas por intermédio da técnica de análise temática, que consistiu em três etapas. A primeira etapa foi a pré-análise, e, mediante uma leitura flutuante, pôde-se fazer formulações e reformulações de hipóteses e objetivos. A segunda etapa foi a exploração do 
material e a terceira etapa foi o tratamento dos resultados obtidos, que foram apresentados na forma de categorias 22 .

A pesquisa foi aprovada pelo Comitê de Ética da Universidade de Fortaleza com parecer número 1.346.007, conforme a resolução número 466/2012, do Conselho Nacional de Saúde, que regulamenta os aspectos éticos legais das pesquisas envolvendo seres humanos ${ }^{23}$

\section{Resultados e discussão}

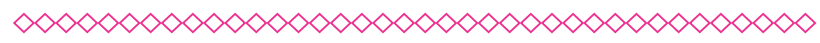

As categorias surgiram mediante as respostas dos pacientes, que foram categorizadas conforme as descobertas, saturação de ideias e significados similares. São elas: descrevendo os benefícios físicos proporcionados pela Fisioterapia; melhorando as relações sociais dos idosos; melhorando os aspectos psíquicos do idoso; a Fisioterapia proporcionando a felicidade.

\section{Descrevendo os benefícios físicos pro- porcionados pela Fisioterapia}

Uma grande aliada no tratamento de disfunções causadas pelo avanço da idade é a Fisioterapia, porque sustenta e acrescenta habilidades para meIhorar sua funcionalidade dentro da capacidade de executar atividades habituais. Na terceira idade, os idosos se limitam a realizarem alguns afazeres em decorrência dessas implicações fisiológicas. Assim, foi analisada a importância da Fisioterapia no controle de alguns desses distúrbios para que os idosos se sentissem capazes de se manterem ativos ${ }^{24}$.

0 treinamento com exercícios funcionais visa à prevenção de comorbidades associadas à saúde do idoso. São movimentos que mobilizam mais de um segmento corporal ao mesmo tempo e que são realizados em diferentes planos, envolvendo diversas ações musculares. Tais exercícios reproduzem ações motoras do cotidiano, sendo este um ponto importante para se investigar os benefícios desse tipo de exercício em idosos ${ }^{25}$.

[...] Me ajudou muito. Melhorou minhas pernas e braços durante essas atividades. Achei legal quando andava no meio da linha. Gostei da bola e dos pesos nas minhas pernas [...]. (Idoso 11).
[...] Me ajudou bastante. Me sinto bem melhor das dores dos braços. Sentia algumas dores antes. Melhorou também minha caminhada. Só não me ajudou mais porque a perna não ajuda também. E aquele exercício de levantar o bastão me ajudou a mexer meus braços bem melhor [...]. (Idoso 1).

[...] Foi bom, com certeza melhorei muito! Consigo mexer melhor os braços. Só a perna que não mexe direito devido ao meu problema que tenho há anos. Mas, tirando isso, a Fisioterapia me ajudou bastante [...]. (Idoso 6).

Percebem-se, nas falas dos idosos, as percepções destes quanto aos benefícios que a Fisioterapia proporcionou durante as atividades realizadas perante suas limitações. Facilitou a execução dos movimentos durante os exercícios. Nota-se, ainda, que houve satisfação e maior participação dos idosos nessas atividades oferecidas no local.

\section{Melhorando as relações sociais dos idosos}

Muitas estratégias de ações que assegurem um envelhecimento saudável e com dignidade vêm sendo implantadas nos países desenvolvidos e em desenvolvimento. Essas ações, muitas vezes, acontecem por meio de programas e grupos sociais para idosos, e possibilitam a socialização, melhoram sua autoimagem perante a sociedade, além de promoverem trocas de experiências que vão favorecer o crescimento mútuo. Para manter o idoso inserido nesses grupos, várias estratégias são desenvolvidas, dentre elas a realização de atividades artesanais, passeios, atividades interativas e atividades físicas, que vão proporcionar troca de experiências, compartilhamento de ideias e reflexão sobre o cotidiano dessas pessoas ${ }^{26}$.

[...] Melhorou, pois a gente vem pra cá e faz os exercícios juntos. É sempre bom quando tem mais gente, assim podemos conversar. Tudo fica animado. E tem os amigos que podem ajudar, se a gente precisar. Eu gosto de fazer a Fisioterapia com mais pessoas. É melhor que fazer só [...]. (Idoso 10).

[...] Melhorou sim, é bom ficar aqui com todos, é bem legal ficar todos juntos, gosto de tá aqui com todos, é mais animado, fica melhor pra gente fazer as atividades porque uns ajudam os outros[...] (Idoso 7). 
[...] Melhorou, sim. É muito bom estar aqui, pois todos aqui são os meus amiguinhos. A gente conversa com os colegas, e os exercícios ficam até mais fáceis de fazer quando estamos todos juntos, pois a gente fica olhando os outros e faz do mesmo jeito [...]. (Idoso 12).

Percebe-se, nas falas dos idosos, a melhoria na sua relação social em razão das atividades realizadas em grupos, visto que estas acabam facilitando a interação entrem eles e possibilitando um meIhor convivio.

Observou-se uma melhor relação social entre os idosos e com os profissionais que prestam assistência a estes, o que proporciona melhor interação e participação durante as atividades oferecidas no local em que residem.

\section{Melhorando os aspectos psíquicos do idoso}

0 isolamento social é um reflexo do tamanho da rede social e escassez de contatos sociais do sujeito. Assim, a atividade grupal destaca-se como valiosa ferramenta para assistir os idosos. A participação em grupos contribui para reduzir o isolamento social e melhorar a adesão às propostas terapêuticas, colaborando para a promoção do envelhecimento ativo e, consequentemente, para a elevação da qualidade de vida de idosos. A prática de atividades que lhes dão prazer e os mantém ativos, são uma forma eficiente de enfrentar as limitações impostas por doenças crônico-degenerativas comuns aos idosos, contribuindo para amenizar o sentimento de solidão ${ }^{27}$.

[...] Ah, eu me sinto muito bem! Me dá muita alegria vir para a Fisioterapia. Tem me ajudado bastante, pois aqui um ajuda o outro. Converso com eles durante as atividades, e isso me dá muito prazer em estar aqui [...]. (Idoso 2).

[...] Eu gosto de tá aqui. Todos são legais com a gente, e isso é de grande alegria para mim. Me sinto bem alegre [...] e satisfeito. A gente fica até feliz de fazer todos os exercícios [...]. (Idoso 9).

[...] Me sinto bem feliz em tá aqui com todos. Fazer as atividades juntos com todos é mais alegre. E sinto até mais animado quando sei que vou para Fisioterapia, pois sei que vou poder fazer atividades, e isso me dá muita alegria [...]. (Idoso 5).

Nas falas dos idosos, constata-se a satisfação psíquica que a Fisioterapia Ihe proporcionou ao realizar os exercícios durante as atividades em grupos. Fica evidente que a participação em grupo melhora o estado psíquico favorecendo a relação entre os participantes, o que torna as atividades mais prazerosas.

\section{A Fisioterapia proporcionando a felici- dade}

Qualidade de vida para os idosos está relacionada ao bem-estar, à felicidade e à realização pessoal. A avaliação dessa qualidade inclui aspectos vinculados as funcionalidades físicas e cognitivas, valores, sentimentos, expectativas ${ }^{28}$. Devem ser empregados métodos científicos pedagógicos e criativos que coloquem o idoso com o corpo em movimento, o cérebro em produção e a sua alma em alegria mediante propostas variadas e diversificadas, abrangendo desde relaxamento, formas estáticas e dinâmicas de equilíbrio e tonicidade, coordenação ${ }^{29}$.

[...] Me sinto bem alegre em fazer $a$ Fisioterapia. Eu fico tranquilo e mais feliz, pois sei que a Fisioterapia vai me ajudar muito a mexer minhas pernas e meus braços [...]. (Idoso 3).

[...] Me sinto feliz em tá aqui com vocês fazendo a Fisioterapia. Me da mais alegria, e posso ficar com meus amigos durante as atividades. Tudo fica mais divertido e animado [...]. (Idoso 8).

[...] Me sinto feliz em fazer a Fisioterapia. É um momento muito alegre pra mim, pois a Fisioterapia me ajuda muito. Eu caminho melhor quando venho pra cá [...]. (Idoso 4).

Observa-se, nas falas dos idosos, que as atividades fisioterapêuticas Ihes melhoram o desempenho físico, facilitando os movimentos; assim, muda o estado emocional, pois está associada à afetividade e à alegria.

Notou-se que a melhoria dos movimentos através das atividades fisioterapêuticas ajudou funcionalmente e emocionAalmente, tornando os idosos mais participativos e proporcionando, de forma geral, uma melhor qualidade de vida. 


\section{Considerações finais}

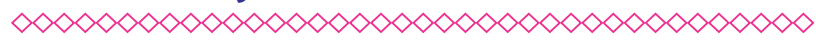

A partir desse estudo, identifica-se que os idosos percebem que as atividades fisioterapêuticas em grupo ajudam nas repercussões motora, sociais e emocionais, uma vez que a interação entre eles favorece o convívio no meio em que residem, visando a incrementar a força muscular, o equilíbrio, a mobilidade, a marcha e o estado emocional, bem como manter o bem-estar físico e social.

Obteve-se uma resposta positiva quanto aos benefícios das atividades fisioterapêuticas em grupo. Além disso, os exercícios possibilitaram a conscientização dos idosos sobre a importância da prática dessas atividades, melhoraram a disposição, animação e interação dos participantes.

Portanto, os idosos perceberam a efetividade da Fisioterapia através das atividades fisioterapêuticas em grupos, visto que estas melhoraram o estado funcional, psíquico e social desse grupo. Este estudo foi importante para sensibilizar os fisioterapeutas que trabalham na área, outros profissionais de saúde, a comunidade acadêmica, os idosos e a população como um todo sobre a relevância das atividades em grupo.

\section{Referências}

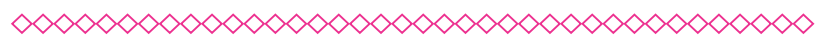

1. Oliveira ECT, Cavalcanti AL. Detecção de risco para (re) hospitalização em idosos: uma revisão sistematizada. Rev. Saúde.Com. 2014; 10(2):209-20.

2. Silva A, Prá KRD. Envelhecimento populacional no Brasil: o lugar das famílias na proteção aos idosos. Argumentum, 2014; 6(1):99-115.

3. Alencar MA, Bruck NNS, Pereira BC, Câmara TMM, Almeida RS. Perfil dos idosos residentes em uma instituição de longa permanência. Rev. Bras. Geriatria Gerontologia. 2012; 15(4):785-96.

4. Menezes JNR, Tomaz SB, Pontes VF, Belchior LD. A autopercepção de idosas sobre o processo de envelhecimento. Estudo. Interdiscipl. Envelhec. 2016; 21(1):135-48.
5. Dawalibi NW, Anacleto WGMC, Witter C, Goulart RMM, Aquino RC. Envelhecimento e qualidade de vida: Análise da produção científica da SCIELO. Estudos de Psicologia. 2013; 30(3):393-403.

6. Zenevicz L, Moriguchi Y, Madureira VSF. A religiosidade no processo de viver envelhecendo. Revista Escola de Enfermagem da Usp. 2013; 47(2):433-9.

7. Marques SR. Tratamento Fisioterapêutico na incontinência urinária em idosas. Revista saúde integrada. 2016; 9(17):110-16.

8. Siqueira AF, Rebesco DB, Amaral FA, Maganhini CB, Agnol SMD, Furmann M, Mascarenhas LPG.

Efeito de um Programa de Fisioterapia Aquática e Capacidade Funcional de Idosos, Revista Saúde e Pesquisa. 2017; 10(2):331-38.

9. Souza CC, Valmorbida LA, Oliveira JP, Borsatto AC, Lorenzini M, Knorst MR, et al. Mobilidade funcional em idosos institucionalizados e não institucionalizados. Rev. Bras. Geriatria Gerontologia. 2013; 16(2):285-93.

10. Santana CS, Bernardes MS, Molina AMTB. Projetos de vida velhice. Estud. interdiscipl. Envelhec. 2016; 21(1):171-86.

11. Fechini BRA, Trompieri N. O Processo de envelhecimento: as principais alterações que acontecem com o idoso com o passar dos anos. Revista Cientifica Internacional. 2012; 1(20):10632.

12. Oliveira JKB, Duarte SFP, Reis LA. Relação entre equilíbrio dados sociodemográfico e conduções de saúde em idosos participantes de grupos de convivência. Estud. interdiscipl. Envelhec. 2016; 21(1):107-21.

13. Fleck CS, Gerzson LR, Steidl SEM, Hernandez NM. Caracterização Da Capacidade Funcional, Nível Cognitivo e Força Muscular Respiratória De Idosas Com Síndrome Parkinsoniana Estud. interdiscipl. envelhec. 2014; 19 (1):109-21.

14. Oliveira JM, Rozendoi CA. Instituição de longa permanência para idosos: um lugar de cuidado para quem não tem opção. Revista Brasileira de Enfermagem. 2014; 67(5):773-9. 
15. Martins E. Constituição e significação de família para idosos institucionalizados: uma visão histórico-cultural do envelhecimento. Estudos e Pesquisas em Psicologia. 2013; 13(1):215-36.

16. Mendes RS, Noveli MMPC. Perfil cognitivo e funcional de idosos moradores de uma instituição de longa permanência para idosos. Cad. Ter. Ocup. UFSCar. 2015; 23(4):723-31.

17. Hoeprs NJ, Ooliveira ACC, Schwalm MT, Soratto MT, Ceretta LB. Medidas de independência funcional em uma instituição de longa permanência. Revista Estudos Interdisciplinares sobre o Envelhecimento. 2013; 18(1):7-26.

18. BARROS TVP, Santos ADB, Gonzaga JM, Lisboa MGC, Brand C. . Capacidade funcional de idosos institucionalizados: revisão integrativa. ABCS Health Sci. 2016; 41(3):176-80.

19. Duarte FM, Araújo KA, Oliveira ES, Lima MPSS. A Importância Da Fisioterapia Na Promoção da Qualidade de Vida Para os Idosos. Caderno de Ciências Biológicas e da Saúde. 2013; 3(1):23-29.

20. Gomes ARL, Campos MS, Mendes MRP, Moussa L. A influência da fisioterapia, com exercícios de equilíbrio, na prevenção de quedas em idosos. FisiSenectus. Unochapecó 2016; 4(1):4-11.

21. Paulo T RS, Castellano WLM, Campos DR, Campos LAS. 0 exercício físico funcional para idosos institucionalizados: um novo olhar para as atividades da vida diária. Revista Estudos Interdisciplinares sobre o Envelhecimento. 2012; 17(2):413-27.

22. MINAYO, MC de S. (Org.). Pesquisa social: teoria, método e criatividade. 23. Ed. Petropólis: Vozes, 2006.
23. BRASIL. Resolução $n^{\circ} 466$, de 12 de dezembro de 2012. Aprovar diretrizes e normas regulamentadoras de pesquisa envolvendo seres humanos.

24. Medeiros JJ, Brito MVG, Perracinni MR, Araújo FB, Santos AD. Aplicabilidade de hidroginástica e musculação em pessoas idosas da comunidade. Revista Interfaces: Saúde, Humanas e Tecnologia. 2014; 2(6):1-4.

25. Gimenes C, Arca EA, Paulino MA, Nicolau NV, Buitoni B, TP Pontes et al. Capacidade funcional de idosas hipertensas submetidas a programa de Fisioterapia funcional. Revista Kairós Gerontologia. 2014; 18(1):77-92.

26. Oliveira DAS, Nascimento JRA Júnior, Bertolini SMMG, Oliveira DV. Participação de idosas em grupos sociais: qualidade de vida e capacidade funcional. Revista Rene. 2016; 17(2):778-84.

27. Paula GR, Souza BN, Santos LF, Barbosa MA, Brasil VV, Oliveira LMAC. Quality of life assessment for health promotion groups. Rev Bras Enferm. 2016; 69(2):242-49.

28. Visentin A, Mantovani MF Caveião C, Mendes TA, Neves AS, Hey AP. Qualidade de vida de idosas hipertensas de uma instituição de longa permanência. Rev Rene. 2015; 16(2):218-25.

29. Banzatto S, Alves AGRC, Silva CM, Viana MO, Freitas IMP, Menezes JNR. Análise da efetividade da Fisioterapia através da psicomotricidade em idosos institucionalizados. Rev Bras Promoção Saúde. 2015; 28(1):119-25. 